\title{
High tempo music prolongs high intensity exercise
}

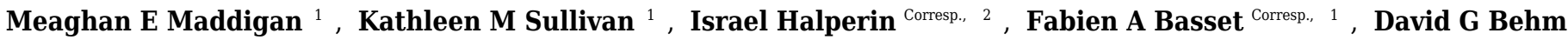 \\ ${ }^{1}$ School of Human Kinetics and Recreation, Memorial University of Newfoundland, St. John's, Newfoundland, Canada \\ 2 School of Human Kinetics and Recreation, Memorial University of Newfoundland, St.John's, Newfoundland, Canada \\ Corresponding Authors: Israel Halperin, Fabien A Basset \\ Email address: Israel.halperin@mun.ca, fbasset@mun.ca
}

Music has been shown to reduce rating of perceived exertion, increase exercise enjoyment and enhance exercise performance, mainly in low-moderate intensity exercises. However, the effects of music are less conclusive with high-intensity activities. The purpose of this with-participant design study was to compare the effects of high tempo music (130 bpm) to a no-music condition during repeated high intensity cycling bouts $(80 \%$ of peak power output (PPO)) on the following measures: time to exercise end-point, rating of perceived exertion (RPE), heart rate (HR), breathing frequency, ventilatory kinetics and blood lactate (BL). Under the music condition, participants exercised $10.7 \%$ longer ( $p=0.035$; Effect size $(E S)=0.28)$ (increase of one minute) and had higher HR $(4 \% ; p=0.043 ; E S=0.25)$, breathing frequency $(11.6 \% ; p<0.001 ; E S=0.57)$, and RER ( $7 \%$ at TTF; $p=0.021 ; E S=1$ .1) during exercise, as measured at the exercise end-point. Trivial differences were observed between conditions in RPE and other ventilatory kinetics during exercise. Interestingly, five minutes post-exercise termination, HR recovery was $13.0 \%$ faster following the music condition $(p<0.05)$ despite that music was not played during this period. These results strengthen the notion that music can alter the association between central motor drive, central cardiovascular command and perceived exertion, and contribute to prolonged exercise durations at higher intensities along with a quicken HR recovery. 


\title{
HIGH TEMPO MUSIC PROLONGS HIGH INTENSITY EXERCISE
}

\author{
Authors: $\quad$ Meaghan E. Maddigan, Kathleen M. Sullivan, Israel Halperin, Fabien A. Basset, \\ David G. Behm \\ Institution: School of Human Kinetics and Recreation \\ Memorial University of Newfoundland \\ St. John's, Newfoundland, Canada
}
Corresponding Authors:
Dr. Fabien A. Basset
School of Human Kinetics and Recreation
Memorial University of Newfoundland
St. John's, Newfoundland, Canada
fbasset@mun.ca
Tel (709) 864-6132
Fax: (709) 864-3979

Running Title: Effect of Music on Exercise

\section{ABSTRACT}

Music has been shown to reduce rating of perceived exertion, increase exercise enjoyment and enhance exercise performance, mainly in low-moderate intensity exercises. However, the effects of music are less conclusive with high-intensity activities. The purpose of this with-participant design study was to compare the effects of high tempo music $(130 \mathrm{bpm})$ to a no-music condition during repeated high intensity cycling bouts $(80 \%$ of peak power output (PPO)) on the following measures: time to exercise end-point, rating of perceived exertion (RPE), heart rate (HR), breathing frequency, ventilatory kinetics and blood lactate (BL). Under the music condition, participants exercised 10.7\% longer $(p=0.035$; Effect size $(E S)=0.28)$ (increase of one minute) and had higher HR $(4 \% ; p=0.043 ; E S=0.25)$, breathing frequency $(11.6 \% ; p<0.001 ; E S=0.57)$, 
34

and RER (7\% at TTF; $p=0.021 ; \mathrm{ES}=1.1)$ during exercise, as measured at the exercise end-point. Trivial differences were observed between conditions in RPE and other ventilatory kinetics during exercise. Interestingly, five minutes post-exercise termination, HR recovery was $13.0 \%$ faster following the music condition $(\mathrm{p}<0.05)$ despite that music was not played during this period. These results strengthen the notion that music can alter the association between central motor drive, central cardiovascular command and perceived exertion, and contribute to prolonged exercise durations at higher intensities along with a quicken HR recovery.

\section{INTRODUCTION}

Music has long been thought to affect the senses (Szmedra and Bacharach 1998) and can act as dissociation during exercise and thus enhance exercise enjoyment and performance (Karageorghis and Priest 2012A/B; Karageorghis, 2016). Music is able to promote ergogenic and psychological benefits during exercise due to three proposed explanations (Karageorghis and Priest 2012A/B; Karageorghis, 2016). First, music may allow individuals to separate thoughts from feelings. This divergence can change one's perception of unpleasant feelings, narrowing the performer's attention and reducing the sensations of fatigue during exercise (Atkinson et al. 2004; Edworthy and Waring 2006; Yamashita et al. 2006; Murrock and Higgins 2009). Second, the divergent stimulus (i.e. music) can alter psychomotor arousal (movement or muscular activity associated with mental processes) and therefore can act as either a stimulant or a sedative prior to and during physical activity (Bigliassi et al, 2018; Carmichael et al. 2018; Szmedra and Bahanach 1998; Yamamoto et al. 2003; Schücker et al. 2009). The third explanation postulates that during continual submaximal activity, an individual is predisposed to respond to rhythmical elements (Nikol et al 2018; Terry et al. 2012; Waterhouse et al. 2009); the result being synchronization between the tempo and the performer's movement making physical activity or 
57 exercise a more harmonious or less stressful experience (Nikol et al 2018; Rendi et al. 2008;

58 Waterhouse et al. 2009).

59

60

61

62

63

64

65

66

67

The available evidence on this topic is congruent and demonstrates that music can and does have a consistent and measurable effect on attention, the ability to trigger a range of emotions, affect mood, increase work output, and encourage rhythmic movement (Nikol et al 2018; Bigliassi et al, 2018; Karageorghis et al, 2008; Scherer 2004; Terry and Karageorghis, 2011). The 'psychophysical' effects primarily examine the perception of effort which in almost all cases involves the Borg's Ratings of Perceived Exertion scale (RPE) (Borg, 1982). These effects are consistent for low and mild intensity activities (Nikol et al. 2018; Terry and Karageorghis 2011) and mostly consistent with high intensity activities (Moss et al. 2018; Haluk, Turchian, and Adnan, 2009).

The effects of music on exercise in the low-to-moderate range of exercise intensities are well established (Karageorghis and Priest, 2012A; Copeland and Franks, 1991; Elliott, Carr, Savage, 2004). Since very high exercise intensities are affected to a high degree by muscle metaboliteinduced failures (peripheral fatigue), there is a lesser influence of the central nervous system (central fatigue) compared to lower exercise intensities (Rejeski, 1985; Tenenbaum et al., 2004). However, it has been shown that peripheral fatigue alone is not able to explain the fatigue induced with higher intensity exercise (Noakes and Gibson, 2004; Noakes, 2012). While fewer studies examined the effect of music on higher intensity exercise, the positive effects seem to persist. For example, listening to music during the Wingate test led to significant improvements in peak power output and decreases in fatigue index with the use of music (Brohmer and Becker 2006; Haluk et al., 2009). This findings are not fully consistent with the perspective of some researchers, suggesting that the 'distraction effect' of music is attenuated at higher exercise 
80 intensities $\left(>70 \%\right.$ maximal oxygen uptake $\left.-\mathrm{VO}_{2 \max }\right)$ due to the internal feedback dominating the

81 capacity of the respective afferent nervous system (Karageorghis et al. 2011). More importantly,

82 it highlights some gaps in the literature with regards to the so-called intensity limitations of

83 music's benefits and the actual mechanisms that result in music's ergogenic effects on exercise

84 performance. Further research is still necessary in order to draw decisive conclusions.

85 Therefore, the primary goal of this study was to examine if listening to high tempo music

86 (130 bpm) while performing high intensity cycling bouts would prolong participant's exercise

87 duration and positively effect common physiological measures of fatigue.

88

89

90

91

92

93

94

95

96

97

\section{METHODS}

\section{Participants}

A convenience sample of sixteen healthy and recreationally active individuals (Table 1) volunteered from the university community to participate in a counterbalanced randomized cross-over design study consisting of a preliminary testing session and two experimental sessions separated by a minimum of two days. Recreationally active was defined as an individual who over the last year did not compete in a structured athletic league (i.e. not a varsity athlete) and was active 1-3 times per week for at least 20 minutes. All the participants filled out a Physical Activity Readiness Questionnaire from the Canadian Society for Exercise Physiology (Canadian Society for Exercise Physiology, 2012) to determine physical activity level and to screen for a history of cardiovascular, pulmonary, metabolic and orthopedic conditions. Informed and written consent was sought from study participants. Participants were blind to the hypotheses of the study. The Memorial University of Newfoundland Human Investigations Committee approved the study [IRB approval number: 11.26].

\section{Experimental Design}


103

104

105

106

107

108

109

110

111

112

113

114

115

116

117

118

119

120

121

122

123

124

125

\section{Preliminary testing session}

Upon the completion of questionnaire filling and anthropometric measurements, participants performed a ramp protocol starting at 50 watts at a self-selected cadence $(>60$ revolutions per minute - RPM) with increment of 1 watt every 3 second to determine $\mathrm{VO}_{2 \max }$ and peak power output (PPO). The test was terminated when the participants reached one of the following criteria: 1) volitional exhaustion, 2) $\mathrm{RPE}$ value $=20$, or 3) $\mathrm{RPM} \leq 60$. The cadence during the incremental test solely assist with monitoring the exercise termination (Kelly and Basset, 2017;

Rossiter et al. 2006; Swart et al., 2009).

Experimental sessions.

Participants sat on an electronically braked stationary bike with feet secured while wearing an oro-nasal facemask to record cardiorespiratory parameters for the duration of the experiment. The protocol consisted of cycles of 4-min high intensity cycling bouts completed at $80 \%$ of PPO (derived from the preliminary testing session) followed by 2-min of active recovery completed at $40 \%$ of PPO. This cycle continued until they reached the exercise end-point (more details below). The exercise protocol stems from training practice. Cyclists implement high intensity interval training into the training regimen to improve the maximal aerobic capacity. The $2: 1$ work/recovery ratio is extensively used by the cycling / running communities and targets the development of endurance characteristics (Bompa and Haff, 2009)

Every minute during the 4-min of the high intensity cycling bout, participants were asked to rate their perceived exertion (RPE) on the Borg scale, $(6-20)$. After each 4-min high intensity cycling bout, $\mathrm{HR}$ and $\mathrm{BL}$ were recorded while seated on the cycle ergometer. HR was continuously recorded throughout the session using a Polar® HR monitor. Upon exercise termination, four of the five measurements (minus blood pressure) were repeated (See 
126 description below). The experimental sessions lasted approximately $2 \mathrm{~h}$ each with the ambient

127 conditions being similar across days $24.0(0.79){ }^{\circ} \mathrm{C}$ and $24.0(0.5){ }^{\circ} \mathrm{C}$, and $1012.75(9.7)$ millibar

128 and 1015.70 (8.8) millibar for Music and No Music, respectively. Participants were then asked to

129 remain in position and relax for 5-min to ensure proper recording of HR and BL recovery while

130 seated on the cycle ergometer. Blood pressure was measured prior to exercise to ensure no

131 participant was hypertensive. Prior to being released from the experiment, an adequate recovery

132 period (resting heart rate $\leq 100 \mathrm{bpm}$ ) was given to all participants.

\section{Instrumentation}

134 Cardiorespiratory Measurements. Oxygen uptake $\left(\mathrm{VO}_{2}\right)$, carbon dioxide output $\left(\mathrm{VCO}_{2}\right)$, 135 breathing frequency and tidal volume were continuously collected with an automated breath-by-

136 breath system (Metamax, Sensor Medics ${ }^{\circledR}$ version Vmax ST 1.0) using a nafion filter tube and a

137 turbine flow meter (opto-electric). Respiratory exchange ratio (RER) and minute ventilation (VE)

138 were calculated as the quotient of $\mathrm{VCO}_{2}$ on $\mathrm{VO}_{2}$ and as the product of breathing frequency by

139 tidal volume, respectively. HR values were transmitted with a Polar HR monitor (PolarElectro,

140 Kempele, Finland). Prior to testing, gas analyzers and volume were calibrated with medically

141 certified calibration gases $\left(16.0 \% \mathrm{O}_{2}\right.$ and $\left.3.98 \% \mathrm{CO}_{2}\right)$ and with a three-liter calibration syringe.

142 In addition, a propane gas calibration was performed to assess the sensitivity of the oxygen and

143 carbon dioxide analysers.

144 Lactate Measurements. All lactate measurements were taken using the Lactate Pro (LP, 145 Arkray KDK, Japan) hand-held portable analyzer. A blood sample of $\geq 5 \mu \mathrm{L}$ was taken from the 146 participant's fingertip using a spring loaded lancet and then blood lactate values were recorded.

147 The company supplied a check strip to confirm that the analyzer operated correctly, and a 
148 calibration strip that provided a non-quantitative indication of analyzer accuracy, which was used

149 at the beginning of each testing session to ensure validity of the measures.

150 Cycle Ergometer. All exercise protocols were performed on an electronically braked

151 Velotron Dynafit Pro cycle ergometer (RacerMate, Inc., Seattle, WA). Factory calibration of the

152 cycle ergometer was performed using Velotron CS software (RacerMate, Inc.) and the Accuwatt

153 rundown verification procedure. Individual positional adjustments (saddle and handlebar height)

154 were made before the first exercise test and were replicated for all subsequent exercise tests.

155 Visual feedback of pedalling rate (RPM) was available on the computer monitor to the

156 participants during each exercise session to allow them to monitor intensity and possibly prolong

157 the exercise duration.

158 Music. All participants listened to the exact same playlist of instrumental popular music

159 which was set to a $130 \mathrm{bpm}$ tempo; meaning each song was not originally $130 \mathrm{bpm}$ but was

160 altered to keep a consistent tempo throughout the playlist. We decided on 130 bpm based on

161 previous studies finding that faster tempos lead to superior physical performance (Waterhouse et

162 al. 2009) and more positive effects compared to slower tempo music conditions (Edworthy and

163 Waring, 2006). Music was played through an Ipod® nano using 'earbud' type head phones and

164 volume was held constant for each participant at 50\% of the maximum volume approximately 65

165 decibels, based on manufacturer specifications of maximum volume being 130 decibels.

166 Exercise Bouts. Participants were given an explanation of the 6-20 point Borg RPE scale and 167 told that if at any time they wished to stop exercising they could do so. After participants were 168 fitted with an armband that held an Ipod(C) nano and ear bud type headphones (headphones and 169 armband were worn regardless of condition), the facemask and mesh headpiece were secured 
170 next and hooked up to the indirect calorimetric system. Each participant then had a 5-min warm

171 up period where they were instructed to keep a cadence of $60-70 \mathrm{rpm}$ for the duration of the

172 exercise protocol, a parameter displayed on a large computer screen. In both music and no music

173 conditions, the experimental sessions started at 40\% of PPO (active recovery), followed by a 4-

174 min high intensity cycling bout at $80 \%$ of PPO. The 2:1 ratio was chosen to ensure that

175 participants were able to complete sufficient numbers of exercise bouts and to accumulate

176 enough exercise time for the subsequent analyses. The 2:1 ratio mimics what well-trained

177 cyclists implement in their interval training program (e.g., Fartlek design). Participants were

178 asked to report the RPE score every minute of the 4-min cycling bout. In addition, at the end of

179 the 4-min high intensity cycling bout BL was assessed. Participants repeated the cycle [4-min

180 work load and 2-min active recovery] until reaching one of the following criteria: 1) volitional

181 exhaustion, 2) $\mathrm{RPE}$ value $=20$, or 3$) \mathrm{RPM} \leq 60$. At the precise exercise end-point, time elapsed

182 from the start of the cycling bouts and final RPE values were recorded followed by final BL

183 sample. To avoid confusion, note that for subsequent analyses the exercise duration did not

184 include time spent in active recovery period.

\section{Data Reduction}

All data sets were analysed using Sigmaplot (version 10.0; Systat Software Inc). First, cardiorespiratory parameters of the incremental test and of the high intensity cycling bouts were smoothed using second-order polynomial function to determine $\mathrm{VO}_{2}$, and its corresponding values of $\mathrm{VCO}_{2}$, breathing frequency, and tidal volume. Second, HR was time-aligned with the scores were interpolated to produce a continuous linear even data point distribution using a twodimensional interpolation function and were then time-aligned as above-mentioned. Fourth, the 
193 high intensity cycling bout epochs were summed to represent time-to-fatigue. Finally, all above

194 time-aligned parameters were expressed in a relative form to correspond to 25, 50, 75 and $100 \%$

195 of total exercise duration. This strategy was decided upon because the exercise durations of each

196 participant were unknown prior to exercise and expected to differ from one participant and

197 condition to the other. The expression of work intensity in a relative form has definitive merits

198 because it provides the possibility of comparing groups with very different characteristics

199 (Basset and Boulay; 2000 and 2003). So, to permit comparison between participants and

200 conditions, we express time in $\%$ of total exercise time to reduce inter-subject variability

\section{Statistical Analysis}

All statistical analyses were conducted using Jamovi (version 0.8). Differences between

music and no-music conditions in time to task failure (measured in seconds and excluding recovery period) were examined using paired t-test. Cardio-respiratory parameters $\left(\mathrm{Bf}, \mathrm{V}_{\mathrm{T}}, \mathrm{V}_{\mathrm{E}}\right.$, $\mathrm{VO}_{2}, \mathrm{VCO}_{2}$ ) and rating of perceived exertion were analyzed with a 2-way ANOVA (2 conditions x 4 times $[25 \%, 50 \%, 75 \%$ and $100 \%$ of total exercise duration]). Differences between conditions in blood lactate were measured with a 2-way ANOVA (2 conditions x 3 time [pretest, immediate post-test, 5-min post-test]). HR was analyzed first with a 2-way ANOVA (2 conditions $\mathrm{x} 4$ time $[25 \%, 50 \%, 75 \%$ and $100 \%$ of time of failure $])$. Second, the effect of music upon HR recovery at the same absolute time period for all participants (pre-test and 5 min posttest) was analyzed with paired t-tests. Gender was treated as a between-subjects variable in all

212 tests. Differences were considered significant at $\mathrm{p}<0.05$. If significant main effects or 213 interactions were present, a Bonferroni (Dunn) procedure was conducted. Cohen $d$ effect sizes

214 were also calculated using the following equation $\left(d=\frac{\text { Mean differences }}{\text { SD average }}\right)$ in which SD average is 
$215 \frac{\sqrt{\text { SD condition } 1+\text { SD condition } 2}}{2}$ to provide qualitative descriptors of standardized effects using these

216 criteria: trivial $<0.2$, small $0.2-0.5$, moderate $0.5-0.8$, and large $>0.8$ (Cohen, 1988). Note that

217 since each participant exercised to volitional fatigue, each testing session was a different length

218 of time; therefore, some variables were collapsed over time or reported as a percentage of TTF.

219 RESULTS

\section{Total Exercise Duration}

The average exercise duration in the music condition $(10: 30 \pm 3: 38$ mins:secs) was $10.7 \%$ longer than the average TTF in the no-music condition $(9: 33 \pm 3: 42$ mins:secs $)(p=0.035 ; \mathrm{ES}=$

(Figure 1A). Therefore on average participants in the music condition completed approximately

2.6 intervals and exercised for one minute longer while participants in the no-music condition only completed approximately 2.3 intervals.

227

228

229

230

231

232

233

234

235

236

\section{Rate of Perceived Exertion (RPE)}

Small magnitude and non-statistically significant $(\mathrm{p} \geq 0.26, \mathrm{ES}=\sim 0.15)$ differences were observed between conditions in absolute RPE across the four time points (Figure 1B). A main effect for time was identified in which RPE increased over the course of the exercise across both conditions $(\mathrm{p}<0.001)$.

\section{Blood Lactate (BL)}

While no statistical interactions or main effect for conditions were observed between conditions at any time point $(\mathrm{p}>0.278)$, the average BL levels post-exercise in the music condition at the exercise end point exhibited a moderate magnitude $12.5 \%$ higher blood concentration than those at post-exercise in the no-music condition $\left(13.5 \pm 2.7 \mathrm{mmol} \cdot \mathrm{L}^{-1} \mathrm{vs} .12 .0\right.$ 
$\left.237 \pm 3.0 \mathrm{mmol} \cdot \mathrm{L}^{-1} ; \mathrm{ES}=0.48\right)($ Figure $1 \mathrm{C})$. These differences likely stem from the longer duration

238 of high intensity activity performed by participants under the music condition.

\section{Heart Rate (HR)}

240 First, a small magnitude and statistically insignificant $(\mathrm{p}=0.223 ; \mathrm{ES}=0.27)$ difference was

241 identified between conditions at resting baseline (Music: 66 \pm 6 , No-music: $62 \pm 7$ ). Second, a main

242 effect for condition was observed in which HR was 4\% higher in the music condition across the

243 four time points $(\mathrm{p}=0.043 ; \mathrm{ES}=0.25)($ Figure $1 \mathrm{D})$. HR increased in both conditions over time

$244(\mathrm{p}<0.001 ; \mathrm{ES}=1.84)$. At post 5 -minutes HR was $13 \%$ lower in the music condition $(99.6 \pm 7.6$

$245 \mathrm{bpm})$ than in the no-music condition $(112.6 \pm 10.6 \mathrm{bpm})(\mathrm{p}<0.001 ; \mathrm{ES}=1.40)$ (Figure 1D).

\section{$246 \mathrm{VO}_{2}$ and $\mathrm{VCO}_{2}$}

247 Small magnitude and statistically insignificant differences were identified in both $\mathrm{VO}_{2}$ and $\mathrm{VCO}_{2}$

248 between conditions and across time ( $\mathrm{p} \geq 0.192 ; \mathrm{ES} \leq 0.31)$ (Figure $2 \mathrm{~A}$ and $\mathrm{B})$.

\section{Ventilation and Tidal Volume}

250 Small magnitude and statistically insignificant differences were identified in ventilation and tidal

251 volume between conditions ( $\mathrm{p} \geq 0.012 ; \mathrm{ES} \leq 0.27$ ) (Figure $2 \mathrm{C}$ and $\mathrm{E}$ ). A main effect for time was

252 observed in which ventilation increased from beginning to the end of the cycling bout $(\mathrm{p}<0.001$;

$253 \quad \mathrm{ES}=0.91)$.

254

255

256

257

\section{Breathing Frequency (Bf)}

A medium magnitude, statistically significant $(\mathrm{ES}=0.57 ; \mathrm{p}=0.006)$ main effect for condition was observed in which average $\mathrm{Bf}$ was $11.6 \%$ higher in the music condition $(43.2 \pm$ 8.7 breath $\left.\cdot \mathrm{min}^{-1}\right)$ compared to the no-music condition $\left(38.7 \pm 8.2\right.$ breath. $\left.\mathrm{min}^{-1}\right)$ (Figure $\left.2 \mathrm{~F}\right)$. A 
258 large magnitude and statistically significant main effect $(\mathrm{p}<0.001$; ES $=1.17)$ for time was

259 identified in which Bf increased by $23 \%$ over time in both conditions from $34.7 \pm 6.2$

260 breath $\bullet \mathrm{min}^{-1}$ to $45.5 \pm 11.5 \mathrm{breath} \cdot \mathrm{min}^{-1}$.

\section{Respiratory Exchange Ratio (RER)}

A significant interaction was observed between conditions and time $(\mathrm{p}=0.040)$. While post-

263 hoc testing only revealed statistical differences favoring the music condition at TTF $(7 \% ; \mathrm{p}=$

$2640.021 ; \mathrm{ES}=1.1)($ Figure 2D). RER was also 5\% greater (non-significant with a large effect size

265 magnitude) at $75 \%$ of TTF $(\mathrm{p}=0.12 ; \mathrm{ES}=0.85) . \mathrm{RER}$ increased over time across both conditions

266 by $5 \%(\mathrm{p}=0.006 ; \mathrm{ES}=0.78)$.

\section{DISCUSSION}

268 The primary purpose of this study was to determine the effects of listening to high tempo 269 music $(130 \mathrm{bpm})$ on physical performance and acute physiological responses during high 270 intensity interval bouts of cycling. Listening to music led to small increases in total exercise 271 duration, accompanied by an increase in $\mathrm{Bf}, \mathrm{HR}$, as well as a steeper heart rate recovery post272 exercise in the high tempo music condition. Yet, RPE scores (Figure 1, panel B), $\mathrm{VO}_{2}$, and $273 \mathrm{VCO}_{2}$ (Figure 2, panel A and B) were similar between conditions although participants exercised 274 longer and recovered faster in high tempo music condition.

275 A possible explanation for the participant's increased exercise duration while experiencing a 276 build-up of peripheral metabolites (increased BL), albeit still reporting comparable RPE scores, 277 can be attributed to a number of psychological influences music had on participants. These 278 include distraction from the sensation of fatigue (Atkinson et al. 2004; Bigliassi et al. 2018; 279 Edworthy and Waring, 2006), greater arousal and positive affect (Bigliassi et al. 2018; 
280 Carmichael et al. 2018; Yamamoto et al. 2003; Schucker et al. 2009), and better synchronization

281 between music and the motor tasks allowing the activity to be more efficient (Nikol et al. 2018;

282 Rendi et al. 2008; Waterhouse et al. 2009). Furthermore, models that emphasise the role of the

283 brain in exercise performance and regulation have pointed to the lack of clear peripherally based

284 fatigue reasons to explain why participants reach the point of exercise failure (i.e., inability to

285 continue exercising) since not all muscle fibers are recruited at the point of exhaustion (Kayser,

286 2003; Noakes, 2000, 2012). As such, even during high intensity exercise efforts, the brain has a

287 role in regulating effort despite the accumulating metabolites in the muscles (Billaut et al. 2011).

288 Henceforth, the CNS must have some control over exercise performance (Kayser, 2003; Noakes,

$2892000,2012)$.

290

The increased breathing frequency response of our participants implies that central command 291 cardiovascular drive regulates through medullar integration the dual-talk between the 292 sympathetic and parasympathetic nervous systems even with high intensity exercise. Breathing

293 frequency is controlled via the autonomic nervous system, by the respiratory centers located in 294 the medulla oblongata in the lower brainstem, functioning largely below the level of 295 consciousness (Bechbache and Duffin, 1977; Williamson, 2010). Hence, at some point during 296 the cycling intervals, performed with high tempo music, the central neural command seemed to 297 modulate the cardiovascular centres. It is well-known that since the tidal volume plateaus around $29860 \%$ of $\mathrm{VO}_{2 \max }$ any increase in ventilation above this threshold mostly results from breathing 299 frequency (Folinsbee, Wallace, Bedi, and Horvath, 1983; Jensen, Lyager, and Pedersen, 1980; 300 Power, Handrigan and Basset, 2011). However, "it should be clear that no single mechanism can 301 be considered to be the sole mediator of the respiratory response" (Makeita and Duffin, 1995). 302 For instance, ventilatory drives due to central command or mechanoreceptor signals, are 
303 independent of ventilation, and provide feedforward control of ventilation during constant-load 304 exercise (Williamson, 2010). Meanwhile, central command can itself be modified by distractive 305 stimuli (e.g. music; Boutcher and Trenske, 1990). Therefore, in this study attention to the 306 external environment might alter the awareness of physiological sensations bringing about 307 changes in control of ventilation via breathing frequency modulation during high intensity 308 exercise (Williamson, 2010). As a result, the participants breathing frequency was higher during 309 high intensity cycling bouts, which could be an indication of the involvement of the cerebral cortex in cardiovascular control mechanisms (Williamson, 2010). It should be noted that beside the above-mentioned significant changes in ventilatory response and despite a longer TTF, no other cardiorespiratory parameters were altered by the music condition (Figure 2). Although the exact mechanism for this physiological response inherent to these cardiorespiratory responses. Although the exact mechanism for this physiological response is unclear, these findings do support the idea that further research into the underlying cortical modulation mechanisms involved with the beneficial effects of music on exercise duration are warranted.

Similar to most of the current literature on this topic, HR during exercise was only slightly affected by the music. However, there was an anomaly in the present study, which has to do with post-exercise recuperative effects of music. Participants who completed the cycling intervals with music had a steeper HR recovery. There is very little known about the impact of music on post-exercise performance. While music seems to relieve stress and improve affective states in non-exercise settings (Särkämö et al. 2008), participants in this study had the music stimulus removed immediately at the end of high intensity cycling bouts and yet five minutes postexercise HR were significantly lower compared to no-music condition. It is unclear to us why 
326 these large differences occurred. We speculate that music may affect the autonomic nervous

327 system; whereby attention to the external environment seems to reduce the awareness of 328 physiological sensations and negative emotions, which may have triggered a quicker

329 parasympathetic response leading to steeper post-exercise HR recovery. These results further 330 support the theory that the CNS has the ability to control some types of exercise performance and 331 aid in the ability to exercise longer at higher intensities; unfortunately the verification of these 332 theories were not within the scope of this investigation.

Although the research is somewhat conflicting when it comes to measuring the extent to which music can enhance exercise performance at maximal or near-maximal levels, this study demonstrated that listening to high tempo music (via headphones) during high intensity cycling intervals can lead to greater physiological performance without increasing individual's perceived exertion. The ability to alter perception is consistent with a vast majority of the current research, which is focused on the psychological effects of music on exercise, mood, and emotion and affect. It is the 'psychophysical' effects or more prudently the physiological effects that have been noted in this study that should motivate future research endeavours. If music can truly distract or disguise the peripheral signs of fatigue it may increase exercise duration, and perhaps enjoyment and adherence. In view of the results presented here, the central motor drive can be uncoupled from the central cardiovascular command to evoke different circulatory responses (Williamson 2010); indeed, the individuals worked harder while breathing at a higher frequency, and experiencing greater muscle fatigue, all whilst diminishing the feeling of discomfort. The association between central motor drive, central cardiovascular command and perceived exertion was clearly altered by distracting stimuli (high music tempo). 
This study suffers from a number of limitations worthy of discussion. First, we altered the

350

351

352

353

354

355

356

357

358

359

360

361

362

363

364

365

366

367

368

369

370

371

tempo of the music to $130 \mathrm{bpm}$ to all songs in the play list. While this allowed for consistency, it

may have affected the experience of the listeners. However, instrumental music was used and thus minor changes in tempo would not have had as major an impact as altered music with lyrics. Second, the sample size was based on a convenience sample and not on an a-priori power calculation.

\section{CONCLUSIONS}

The music condition in the present study elicited an increase in exercise duration, breathing frequency, and HR while not influencing RPE and other ventilatory kinetics during exercise. Additionally, a steeper heart rate recovery post-exercise compared to the control condition was observed. These results support the notion that music can modify the interplay between central motor drive, central cardiovascular command and perceived exertion. Changes in factors such as breathing frequency suggest that this modification may occur at a subconscious level. Therefore, further studies of the effect of music on exercise performance should focus on the physiological mechanisms responsible for the observed changes, including potentially more complex procedures (i.e. fMRI) as opposed to the psychometric measurements.

\section{REFERENCES}

Atkinson, G., Wilson, D., Eubank, M. 2004. Effect of music on work rate distribution during a cycle time trial. Int. J. Sports. Med. 62: 413-419.

Basset, FA, Boulay, MR (2000). Specificity of treadmill and cycle ergometer tests in triathletes, runners and cyclists. Eur. J Appl. Physiol. 81: 214-221

Basset, F.A., Boulay, M.R. (2003). Treadmill and cycle ergometer tests are interchangeable to monitor triathletes annual training. J. Sports Sci. Med. 2: 110-116 
372 Bechbache, R.R., Duffin, J. (1977). The entrainment of breathing frequency by exercise rhythm.

373 The Journal of Physiology. 272(3): 553-561.

374 Bigliassi, M., Karageorghis, C. I., Hoy, G. K., \& Layne, G. S. (2018). The Way You Make Me

375 Feel: Psychological and cerebral responses to music during real-life physical

376 activity. Psychology of Sport and Exercise (in-press).

377 Bompa, T.O., Haff, G.G. Periodization: theory and methodology of training. Human Kinetics, $378 \quad 2009$

379 Boutcher, S.H., Trenske, M. (1990). The effects of sensory deprivation and music on perceived 380 exertion and affect during exercise. J. Sport. Exerc. Psychol. 12(2): 167-176.

381 Borg, G. A. (1982). Psychophysical bases of perceived exertion. Med sci sports exerc, 14(5), $382 \quad 377-381$.

383 Brohmer R., Becker C. (2006). Wingate performance and music. J. Undergraduate Kinesiol. Res, $3842(1), 49-54$.

385 Canadian Society for Exercise P. The Canadian Physical Activity, Fitness and Lifestyle

386 Approach. Ottawa: Health Canada Publishers, 2012.

387 Carmichael, K. E., Marshall, D. N., Roche, B. M., \& Olson, R. L. 2018. Effects of Music on 388 Arousal, Affect, and Mood Following Moderate-Intensity Cycling. International Journal of 389 Exercise Science: Conference Proceedings. 2 (10) 91-100.

390 Cohen J. 1988. Statistical Power Analysis for the Behavioral Sciences. 2nd ed. Hillsdale (NJ):

391 Lawerence Earlbaum Associates.

392 Copeland, B.L., Franks, B.D. 1991. Effects of types and intensities of background music on 393 treadmill endurance. J. Sports. Med. Phys. Fitness. 31(1): 100-103.

394 Edworthy, J., Waring, H. 2006. The effects of music tempo and loudness level on treadmill 395 exercise. Ergonomics. 49: 1597-1610.

396 Elliott, D., Carr, S., Savage, D. 2004. Effects of motivational music on work output and affective 397 responses during sub-maximal cycling of a standardized perceived intensity. J. Sport. Behav. 398 27(2): 134-147.

399 Folinsbee, L. J., Wallace, E. S., Bedi, J. F., Horvath, S. M. 1983. Exercise respiratory pattern in 400 elite cyclists and sedentary subjects. Med. Sci. Sports Exerc., 15: 503-509.

401 Gandevia, S.C. 2001. Spinal and supraspinal factors in human muscle fatigue. Physiol. Rev. 81: $402 \quad 1725-1789$. 
403 Haluk, Koc \& Turchian, Curtseit \& Adnan, Curtseit (2009-06-01). Influence of music on 404 wingate anaerobic test performance.(SPORT AND PERFORMANCE)(Report). In Ovidius 405 University Annals, Series Physical Education and Sport/Science, Movement and Health. 9 (2), 406 134(4).

407 Jensen, J. I., Lyager, S., Pedersen, O. F. 1980. The relationship between maximal ventilation, 408 breathing pattern and mechanical limitation of ventilation. J. Physiol. 309: 521-532

409 Karageorghis, C.I. Applying music in exercise and sport. Human Kinetics, 2016.

410 Karageorghis, C.I., Jones, L., Stuart, D.P. 2008. Psychological effects of music tempi during 411 exercise. Int. J. Sports. Med. 29: 613-619.

412 Karageorghis, C.I., Priest, D.L. 2012A. Music in the exercise domain: a review and synthesis 413 (Part I). Int. Rev. Sport. Exerc. Psychol. 5(1): 44-66.

414 Karageorghis, C.I., Priest, D.L. 2012B. Music in the exercise domain: a review and synthesis 415 (Part II). Int. Rev. Sport. Exerc. Psychol. 5(1): 67-84.

416 Karageorghis, C.I., Terry, P.C., Lane, A.M., Bishop, D.T., Priest, D.L. 2011. British Association 417 of Sport and Exercise Sciences expert statement on the use of music in exercise. The Sport and 418 Exercise Scientist. 28: 18-19.

419 Kayser, B. 2003. Exercise begins and ends in the brain. Eur. J. Appl. Physiol. 90: 411-419.

420 Kelly, L.P., Basset, F.A. 2017. Acute normobaric hypoxia increases post-exercise lipid oxidation 421 in healthy males. Front. Physiol. (doi: 10.3389/fphys.2017.00293).

422 Mateika, J.H., Duffln, J. 1995. A review of the control of breathing during exercise. Eur. J. Appl.

423 Physiol. 71: 1-27

424 Moss, S. L., Enright, K., \& Cushman, S. (2018). The influence of music genre on explosive 425 power, repetitions to failure and mood responses during resistance exercise. Psychology of Sport 426 and Exercise, 37, 128-138.

427 Murrock, C.J., Higgins P.A. 2009. The theory of music, mood and movement to improve health 428 outcomes. J. Adv. Nurs. 65(10): 2249-57.

429 Nikol, L., Kuan, G., Ong, M., Chang, Y. K., \& Terry, P. C. (2018). The Heat Is On: Effects of 430 Synchronous Music on Psychophysiological Parameters and Running Performance in Hot and 431 Humid Conditions. Frontiers in psychology, 9, 1114.

432 Noakes, T.D. 2000. Physiological models to understand exercise fatigue and the adaptations that 433 predict or enhance athletic performance. Scand. J. Med. Sci. Sports. 10: 123-125. 
434 Noakes, T. D. O. (2012). Fatigue is a brain-derived emotion that regulates the exercise behavior 435 to ensure the protection of whole body homeostasis. Frontiers in physiology, 3, 82.

436 Noakes, T.D., Gibson, A.S.C. 2004. Logical limitations to the "catastrophe" models of fatigue 437 during exercise in humans. Br. J. Sports. Med. 38: 1-30.

438 Power, G.A., Handrigan, G.A., Basset, F.A. 2011. Ventilatory response during an incremental 439 exercise test: A mode of testing effect. Eur. J. Sport Scie. 12: 491-498

440 Rejeski, W. J. 1985. Perceived exertion: an active or passive process? J. Sport. Psychol. 7: 371 441378.

442 Rendi, M.R., Szabo, A., Szaba, T.S. 2008. Performance Enhancement with Music in Rowing 443 Sprint. Sport Psychologist. 22(2): 175-182.

444 Rossiter, H.B., Kowalchuk, J. M., Whipp, B. J. 2006. A test to establish maximum $\mathrm{O}_{2}$ uptake 445 despite no plateau in the O2 uptake response to ramp incremental exercise. J. Appl. Physiol. 446 100:764-770.

447 Särkämö, T., Tervaniemi, M., Laitinen, S., Forsblom, A., Soinila, S., Mikkonen, M., Autti, T., 448 Silvennoinen, H.M., Erkkilä, J., Laine, M., Peretz, I., Hietanen M. 2008. Music listening 449 enhances cognitive recovery and mood after middle cerebral artery stroke. Brain. 131: 866-876. 450 Scherer, K.R. 2004. Which emotions can be induced by music? What are the underlying 451 mechanisms? And how can we measure them? Journal of New Music Research. 33: 239-251. 452 Schücker, L., Hagemann, N., Strauss, B., Völker K. 2009. The effect of attentional focus on 453 running economy. J. Sports. Sci. 27(12): 1241-1248.

454 Swart, J., Lamberts, R.P., Lambert, M.I., Lambert, E.V., Woolrich, R.W., Johnston, S., Noakes, 455 T.D. Exercising with reserve: exercise regulation by perceived exertion in relation to duration of 456 exercise and knowledge of endpoint. Br. J. Sports Med. 2009;43;775-781

457 Szmedra, L., Bacharach, B.W. 1998. Effect of music on perceived exertion, plasma lactate, 458 norepinephrine and cardiovascular hemodynamics during treadmill running. Int. J. Sports. Med. 459 19(1): 32-37.

460 Tenenabum, G., Lidor, R., Lavyan, N., Morrow, K., Tonnel, S., Gershgoren, A., Johnson, M. 461 2004. The effect of music type on running perseverance and coping with effort sensations.

462 Psychol. Sport. Exerc. 5: 89-109.

463 Terry, P.C., Karageorghis, C.I. 2011. Music in sport and exercise. In T. Morris and P.C. Terry 464 (Eds.). The new sport and exercise psychology companion. Morgantown, WV: Fitness 
465 Information Technology. pp. 359-380.

466 Terry, P.C., Karageorghis, C.I., Saha, A.M. and D’Auria, S., 2012. Effects of synchronous music

467 on treadmill running among elite triathletes. Journal of Science and Medicine in Sport, 15(1),

468 pp.52-57.

469 Waterhouse, J., Hudson, P., Edwards, B. 2009. Effects of music tempo upon submaximal cycling

470 performance. Scand. J. Med. Sci. Sports. 20(4), 662-669.

471 Yamamoto, T., Ohkuwa, T., Itoh, H., Kitoh, M., Terasawa, J., Tsuda, T., Sato, Y. 2003. Effects

472 of pre-exercise listening to slow and fast rhythm music on supramacimal cycle performance and 473 selected metabolic variables. Arch. Physiol. Biochem. 111: 211-214.

474 Yamashita, S., Iwai, K., Akimoto, T., Sugawara, J., Kono, I. 2006. Effects of music during

475 exercise on RPE, heart rate and the autonomic nervous system. J. Sports. Med. Phys. Fitness. 46: $476 \quad 425-43$.

477 Williamson, J.W. 2010. The relevance of central command for the neural cardiovascular control 478 of exercise. Exp. Physiol. 95: 1043-1048

\section{Figure captions}

Figure 1. All figures include means \pm SD. B: $R P E=$ Rate of perceived exertion. $\mathbf{C}: \mathrm{BL}=\mathrm{Blood}$ lactate. D: HR= Heart rate. $(*)$ illustrates significant differences between conditions.

Figure 2. All figures include means $\pm \mathrm{SD}$. $\mathbf{A}$ : $\mathrm{VO}_{2}=$ Maximal oxygen uptake. B: $\mathrm{VCO}_{2}=\mathrm{Carbon}$ dioxide output. $\mathbf{C}: \mathrm{V}_{\mathrm{T}}=$ Tidal volume. D: $\mathrm{RER}=$ Respiratory exchange ratio. $\mathbf{E}$ : $\mathrm{VE}=$ Minute ventilation. $\mathbf{F}: \mathbf{B f}=$ Breathing frequency. $(*)$ illustrates significant differences between conditions. 


\section{Table 1 (on next page)}

Subject characteristics.

All data is presented as means and SD. 
1 Table 1. Subject characteristics. All data is presented as means and SD.

2

\begin{tabular}{lccc}
\hline & Males $(\mathbf{n}=\mathbf{8})$ & Females $(\mathbf{n}=\mathbf{8})$ & All $(\mathbf{n}=\mathbf{1 6})$ \\
\hline Age $($ years $)$ & $30.5 \pm 3.7$ & $28.3 \pm 3.4$ & $29.4 \pm 3.6$ \\
Mass $(\mathrm{kg})$ & $75.2 \pm 7.4$ & $65.9 \pm 4.7$ & $70.5 \pm 7.6$ \\
Height $(\mathrm{cm})$ & $178.3 \pm 6.2$ & $164.9 \pm 5.2$ & $171.6 \pm 8.8$ \\
$\mathrm{VO}_{2 \max }\left(\mathrm{L} \cdot \mathrm{min}^{-1}\right)$ & $4.1 \pm 0.4$ & $3 \pm 0.3$ & $3.51 \pm 0.70$ \\
\hline
\end{tabular}

3 
Figure 1

\section{Performance measures}

All figures include means \pm SD. B: RPE $=$ Rate of perceived exertion. C: BL= Blood lactate. $D$ : $\mathrm{HR}=$ Heart rate.
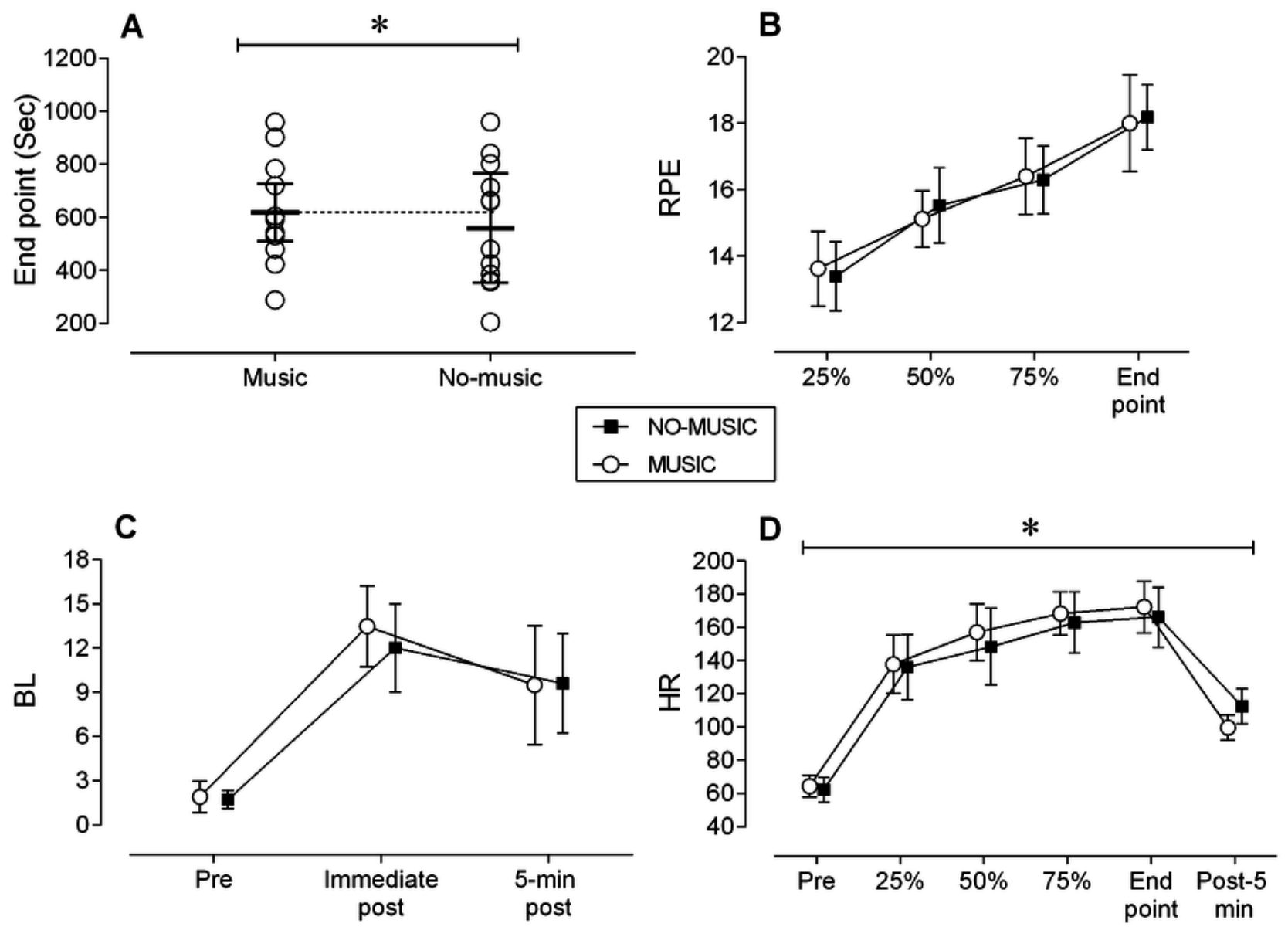
Figure 2

Metabolic data

All figures include means+SD. A: $\mathrm{VO}_{2}=$ Maximal oxygen uptake. B: $\mathrm{VCO}_{2}=$ Carbon dioxide output. $\mathbf{C}: \mathrm{V}_{\mathrm{T}}=$ Tidal volume. $\mathbf{D}: \mathrm{RER}=$ Respiratory exchange ratio. $\mathbf{E}: \mathrm{VE}=$ Minute ventilation.

$\mathbf{F}: \mathbf{B f}=$ Breathing frequency

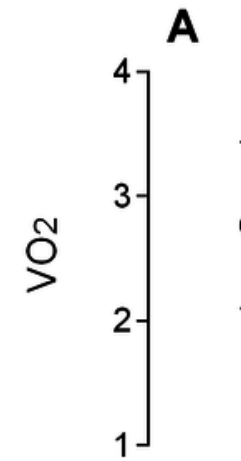

C
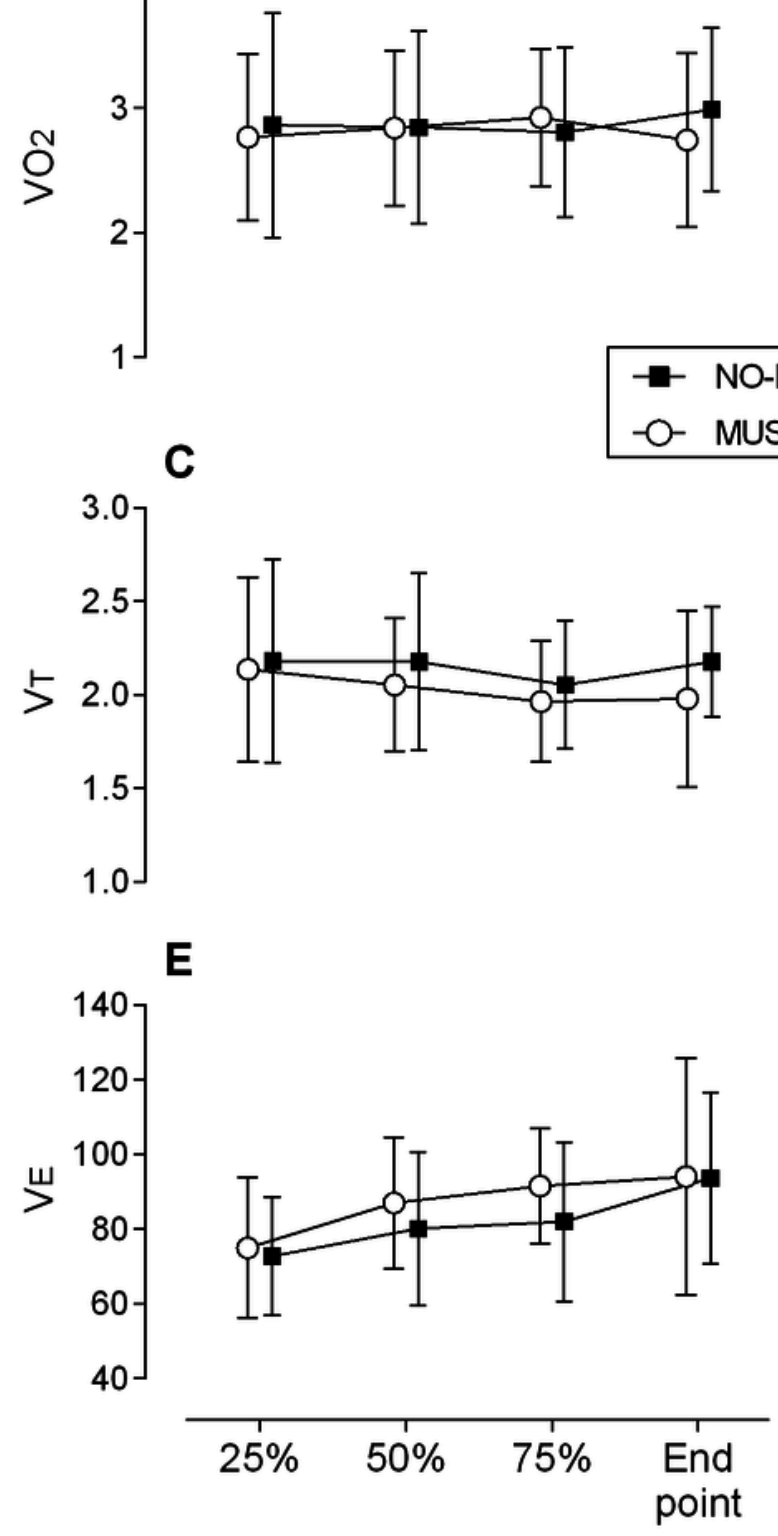

Time
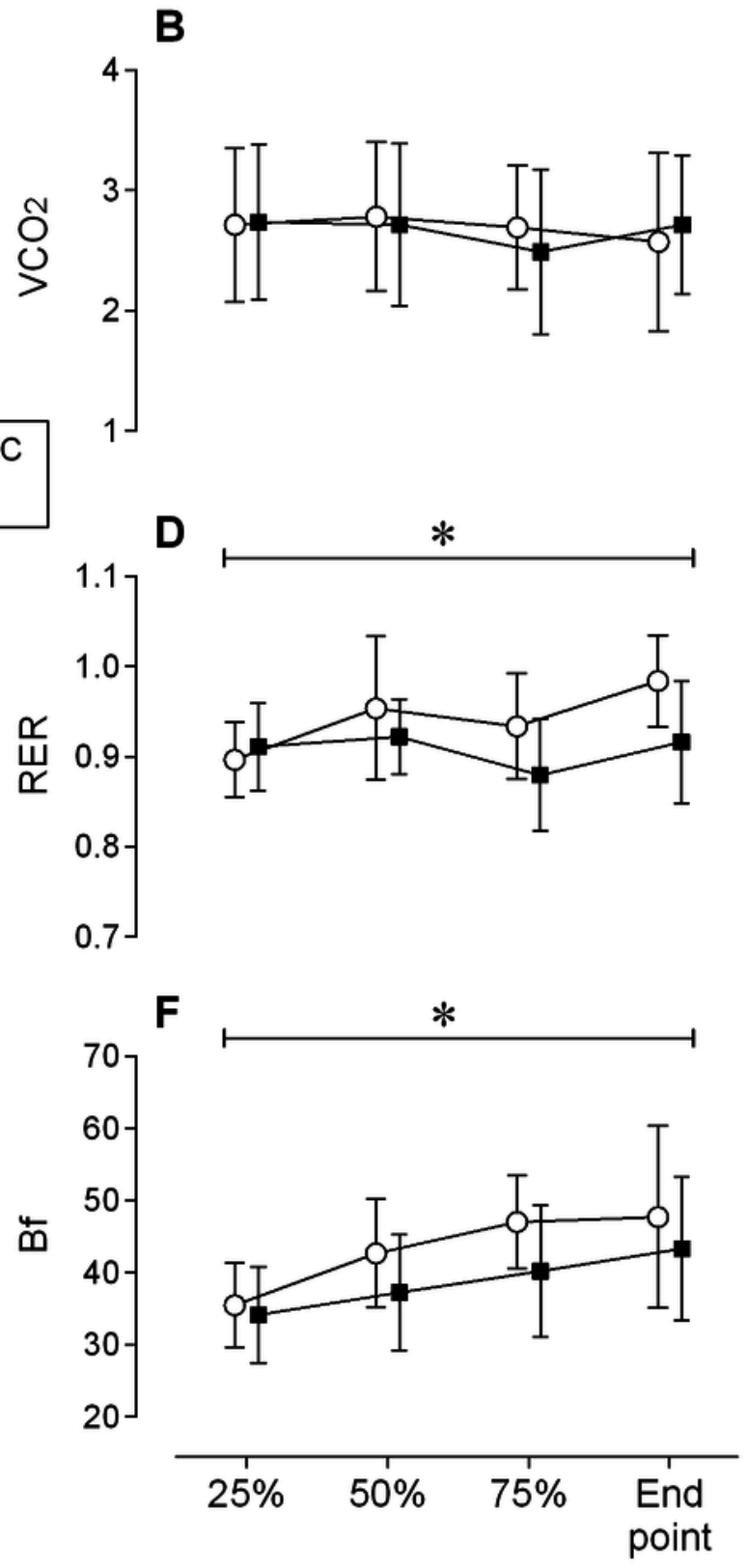

Time 